\title{
Diversidad Familiar, Relaciones de Género y Producción de Cuidados en Salud en el Modelo de Salud Familiar: Análisis de Caso en un CESFAM de la Región Metropolitana, Chile.
}

\section{Family Diversity, Gender Relations and Health Care in the Familiy Health Approach: Case Study in CESFAM in the Chilean Metropolitan Area}

\author{
Irene Magaña \\ Claudia Calquín \\ Sebastián Silva \\ $\mathrm{y}$ \\ Makarena García \\ Universidad de Santiago de Chile, USACH, Chile
}

(Rec: 13 de enero de 2010; Acep: 4 de enero de 2011)

\section{Resumen}

Por medio de este estudio se analiza el papel de las mujeres y de las familias en la producción de los cuidados en salud desde los discursos que producen los equipos de salud y el análisis de las fichas familiares en un CESFAM de una localidad urbano-rural de la Región Metropolitana. Se concluye que a pesar de las reformas modernizadoras impulsadas por el Estado en el sector salud, se perpetúa el lugar tradicional de las mujeres y su condición de género, así como un ideario de familia sustentado en la clásica división sexual del trabajo. Asimismo se aprecia que a pesar de la centralidad del papel de las mujeres en la producción de la salud familiar, este permanece invisibilizado y sólo se transparenta con relación a la responsabilidad de estas en las problemáticas familiares.

Palabras clave: Modelo de Salud Familiar, género, diversidad familiar y organización de los cuidados

\begin{abstract}
We have analyzed the role of women and their families regarding the health group speeches and the analysis of the family records from CESFAM in an urban rural place in the Metropolitan region. It is concluded that in spite of the modern reforms boosted by the state health department the traditional role of women and their genre condition remains the same as well as their way of living supported by the classical sex division in their jobs. At the same time it is observed that in spite of the central role of women in maintaining the family health, this remains unseen and it is only observed in their responsibilities in the family circumstances Key words: Health family model, gender, family diversity and organization of care.
\end{abstract}




\section{Introducción}

Una parte sustantiva de lo que es la actual Reforma de Salud chilena ha consistido en la implementación del Modelo de Salud Familiar, modelo de atención más cercano a las personas y a la comunidad, con un énfasis mayor en el nivel primario y que operativamente se traduce en la conversión de los tradicionales "Consultorios" de atención primaria en "Centros de Salud Familiar" (MINSAL, 2008).

Por tareas de cuidado se entiende las que las mujeres han asumido a lo largo del tiempo a partir de la división sexual del trabajo, en donde la familia ideal se constituía por un hombre proveedor y una mujer ama de casa responsable de la crianza de los hijos y el aseo y cuidado del hogar. Con la incorporación de las mujeres al trabajo laboral remunerado, queda pendiente el cómo resolver las tareas de cuidado de la familia imprescindibles para subsistir. Entre las tareas de cuidado se encuentran el cuidado de los hijos e hijas -la crianza-, el cuidado de los miembros trabajadores del hogar -tradicionalmente el marido-, el cuidado y mantenimiento del hogar, las labores de enlace - pagar cuentas, llevar y traer a los niños y niñas del colegio-, el cuidado de enfermos, tercera edad o discapacitados y las labores relacionadas con -sobre todo en el caso de familias de escasos recursos- el cobro de bonos o subsidios, los controles médicos obligatorios -propios y de los demás-y finalmente el cuidado de sí mismo. Por ello durante el Consenso de Quito (CEPAL, 2007) se dio especial relevancia a la contribución de las mujeres a la economía nacional y, en particular, a la protección social. Estas tareas de cuidado son invisibles estadísticamente, ya que no se cuentan en las cuentas nacionales, tampoco en las tradicionales mediciones económicas como el PIB o el PNB. La única manera de conocer a cabalidad cómo se articula en las sociedades el cuidado es través de encuestas de uso del tiempo, en donde se puede ver cuantas horas dedican hombres y mujeres a las diversas tareas de cuidado no remuneradas.

Este proceso ha exigido un cambio paradigmático en torno a la comprensión de la relación entre los usuarios y las formas de producción de la salud. Cambio que exige visibilizar un nuevo concepto de organización del cuidado de salud en las familias, que se produce en forma desigual y contradictoria tanto en las distintas instancias del sistema de salud, como en las familias mismas, tensionando las relaciones entre las partes y las formas en que se produce el cuidado.

Tal como señala Durán (2005), la producción de salud tiene lugar principalmente en el hogar a través de múltiples tareas de cuidado realizadas tradicionalmente por mujeres, correspondiendo al sistema público o privado de la salud un porcentaje menor, aunque indispensable, de estos cuidados.
Esta noción es aplicada en Chile en el marco del proyecto Equidad de Género y Reforma de la Salud (Gálvez \& Matamala, 2001), a partir del cual el MINSAL reconoce lo doméstico y lo institucional como espacios de cuidado en salud, destacando el papel protagónico de las mujeres en dichos sistemas (MINSAL, 2002).

Otras publicaciones extienden el análisis sobre los cuidados de salud, al aporte no remunerado de las mujeres y las organizaciones comunitarias (Calvin \& Grandón, 1995; Provoste 2002). La Organización Panamericana de la Salud (Reca, Álvarez \& Tijoux, 2008) identifica como parte de las inquietudes de género en salud la carga de trabajo no remunerado que recae sobre las mujeres en el hogar, agregando que los cuidados domésticos de la salud han aumentado en Chile, consecuencia de las últimas reformas en salud, debido a factores como el aumento de la cobertura y el incremento de tratamientos e intervenciones ambulatorias y de enfermedades crónicas y degenerativas que requieren atención en el hogar, como el programa de hospitalización domiciliaria (MINSAL, 2002).

Con relación al reconocimiento social y económico de los cuidados domésticos de salud, la OPS, junto a UNIFEM y CEPAL (2005) han impulsado la realización de Encuestas Nacionales de Uso del Tiempo y la medición del trabajo no remunerado en salud, en cuentas satélites del sector hogares, para su incorporación a las Cuentas Nacionales. Los resultados preliminares obtenidos en la Encuesta Nacional sobre Uso del Tiempo en Chile muestran que nueve de cada diez cuidadores de salud no remunerados son mujeres $(86,4 \%)$ que tienen bajo su responsabilidad la mayoría de las tareas domésticas y de cuidado, así como la organización y funcionamiento del hogar. Además, más de $15 \%$ de ellas tienen la responsabilidad económica del hogar, es decir que ellas son quienes más ingresos aportan al hogar (Hopenhayn, 2009).

Desde esta perspectiva, el modelo de atención puede ser visto como una zona de articulación entre los centros de atención del sistema público y las familias destinatarias de su acción (Berlagoscky \& Provoste, 2002), siendo la forma de esa articulación la clave del éxito o fracaso del modelo. Al respecto, se argumenta que los principales logros sanitarios del siglo XX en Chile como son ejemplo la reducción de mortalidad infantil y materna, la cobertura casi universal de las vacunas y otros se sustentaron en un amplio contingente de mujeres de sectores populares no incorporadas al trabajo remunerado, así como en una alta proporción de familias numerosas y de carácter extenso, que permitían la disponibilidad del tiempo de algunas mujeres de cada hogar para la interacción con los "consultorios". Pero estas condiciones de organización familiar y distribución de roles sexuales ya no existen (Arriagada, 2005; La Parra, 2001).

Frente a esto, resulta entonces relevante cuestionar ¿cómo incorpora el actual modelo de atención, 
especialmente sus equipos de trabajo, los considerables cambios y las nuevas diversidades propias de las familias chilenas?, ¿existen cambios representacionales y culturales en los equipos de salud que permitan interactuar bajo un modelo de salud familiar, con una familia diversa y compleja? y ¿cómo se integran en estas nuevas diversidades familiares los papeles asociados a las actuales configuraciones de género ${ }^{l}$ ?

No es claro que el diseño del Modelo de Salud Familiar haya considerado su adecuación a tales transformaciones. En una primera aproximación podríamos suponer que se están reproduciendo los modelos y concepciones homogéneos y tradicionales de familia, con roles de género clásicamente definidos como usuarios de los centros de salud. Situación en que hoy sólo se encontraría un porcentaje menor de hogares (Berlagoscky \& Provoste, 2002; Rico, 2009). Además, un supuesto semejante afectaría a los equipos de salud, lo que se traduciría en reproducir prácticas dirigidas a un interlocutor que no se encuentra presente, ya que los tradicionales usuarios y usuarias se han transformado, gracias a la diversificación que se produce en las instituciones como la familia.

Así, por una parte, no se aprecia que se tomen medidas especiales para la relación con grupos familiares donde la mujer está trabajando remuneradamente o es jefa de hogar; ni se conoce la forma en que en este nuevo contexto se abordan o reasignan los cuidados de la salud entre los miembros del hogar (Rodríguez, 2005); y por otra parte, la praxis de los equipos de salud perpetúan -en sus imaginarios- esquemas propios de la atención individualizada y centrada en las formas de producción y atención de la enfermedad, más que en la promoción de condiciones para la salud familiar.

Resulta entonces necesario comprender la reforma de salud y en particular la implementación del Modelo de Salud Familiar y cuál sido el impacto de estos cambios en la construcción de imaginarios institucionales de los equipos de salud. Inscritos en el proyecto consecuente del actual modelo propuesto por el Estado, expresado en lineamientos estratégicos como: la modernización administrativa, los procesos de sectorización, la coordinación intersectorial, la descentralización y la participación comunitaria (González \& Flores, 2008; Infante, Mata \& López-Acuna, 2000; Mesa-Lago, 2005; Ugalde \& Homedes, 2002). Medidas de ajuste que ubican al sector salud en el camino del recorte presupuestal, la recomposición del gasto sectorial, y la privatización de áreas y sectores de atención, antes de responsabilidad estatal (Brito, 2000; Di Gropello \& Cominetti, 1998; González \& Flores 2008).

Con el objetivo de contribuir al conocimiento sobre los desfases y tensiones del Modelo de Salud implementado por

Entre estos se destaca la incorporación de las mujeres al trabajo remunerado y aumento de las jefaturas de hogar femeninas, reflejados en el Censo 2002 y en diversos estudios. Véase por ejemplo, de Irma Arriagada (2005). los CESFAM y las familias usuarias, a la luz de las nuevas organizaciones familiares, se indaga desde los discursos de un equipo de salud en la construcción subjetiva en torno a las prácticas que sustentan la relación del Equipo del CESFAM y las familias usuarias, accediendo a sus representaciones de la organización de las familias consultantes, de las principales problemáticas de salud familiar y de las tareas de cuidado que ejercen hombres y mujeres al interior de los hogares.

\section{Método}

La presente investigación, de carácter empírica, exploratoria y comprensiva, se realizó utilizando como metodología de investigación al "estudio de caso", ya que como "investigación micro-social” (Jelin, Llovet \& Ramos, 1999, citado en Olavarria, 2001) permite estudiar un fenómeno contemporáneo (en el que los límites entre el fenómeno y el contexto no son claramente visibles) desde su contexto real, y utilizando distintas fuentes de evidencia. De hecho, siguiendo el propósito de esta herramienta de investigación, se trató de buscar ciertos patrones y consistencias en la interacción entre las distintas partes del sistema elegido como caso, por medio del empleo de técnicas como entrevistas grupales, entrevistas individuales semi-estructuradas a informantes claves y el estudio de fuentes secundarias, para realizar un análisis que sea susceptible de ser aplicado de manera genérica para la comprensión de nuestra proposición teórica.

Como caso único, se eligió un CESFAM que por intención guiada teóricamente, se caracteriza por estar en una zona urbano-rural, en la que parte de su población está ligada a actividades de pequeña agricultura, pero que actualmente está fuertemente mezclada con una población migratoria del sector empobrecido de la Región Metropolitana, dedicada al trabajo en industrias y al sector de la construcción. Es por tanto una localidad que reúne características de la diversidad familiar producto de su condición urbano-rural y la composición demográfica que esto significa.

La investigación general tuvo tres fases de trabajo. Se inició con el estudio de fuentes secundarias donde se revisaron la totalidad de las fichas familiares del CESFAM (descartando las fichas personales y/o las que no presentaban información sobre el grupo familiar). A partir de estos datos se crearon tipificaciones relativas a la caracterización de la organización del sistema familiar consultante, las organizaciones de estructura familiar y estructura de cuidados, las problemáticas de salud en la familia, tipos y tareas de cuidado y motivos de consulta.

En base a estas primeras tipificaciones, se construyó una pauta para las entrevistas grupales con los equipos de salud (tanto del estamento de funcionarios como del profesional) y para las entrevistas semi-estructuradas con los informantes claves. 
En una segunda etapa se realizaron las entrevistas grupales, considerando a la totalidad de los integrantes de los equipos del CESFAM, y en una tercera, las entrevistas a informantes claves (directora del CESFAM, asistente social, médico general, enfermera).

Este material se analizó de dos formas. Primero aplicando el programa informático MAXQDA, el cual permite realizar un análisis descriptivo de categorización abierta y relacional de datos. Segundo, elaborando una triangulación de la información, según lo obtenido en: el análisis de las fichas familiares, las entrevistas grupales y las entrevistas a informantes claves.

Finalmente, se realizó un análisis interpretativo de todos los materiales recogidos en relación con cada una de las categorías levantadas, tanto en la categorización abierta y relacional, como en la triangulación de los datos de las tres fuentes investigadas.

En todas las etapas se observaron normas éticas como consentimiento informado de los participantes y confidencialidad de la información.

\section{Resultados}

La triangulación de la información dio lugar a una categorización abierta que se organizó en torno a dos esquemas relacionales. El primero da cuenta de las organizaciones familiares y las demandas de atención. El segundo de la relación de los Cuidados domésticos en salud y de las percepciones de los equipos de salud respecto de la organización y cuidados en las familias.

Figura 1:

Familias y Demandas en Atención Primaria

[Según entrevistas grupales a equipo de salud, entrevistas individuales a informantes claves y datos de las fichas de las familias usuarias]

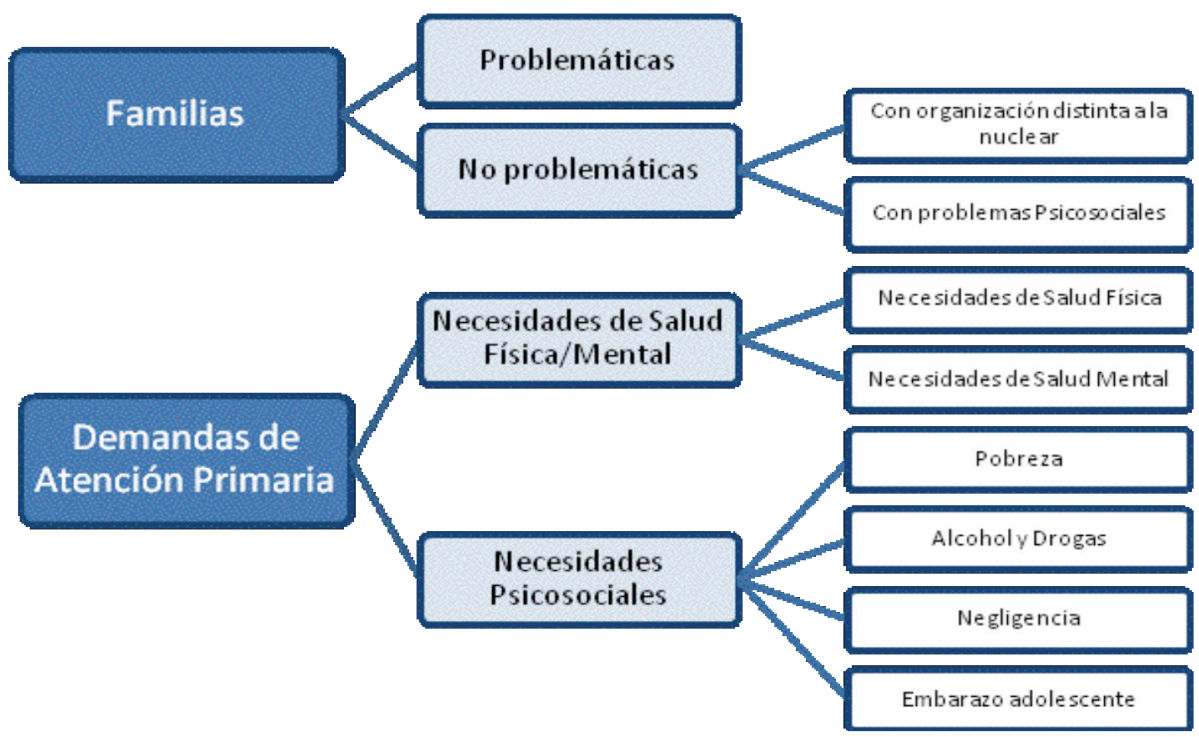

1. Familias.

La categoría Familia oscila entre las ideas de Familias Problemáticas y Familias no problemáticas y/o funcionales.

\subsection{Familias No-Problemáticas:}

Se representan estructuras familiares por la negación de estructuras no tradicionales y "perturbaciones" asociadas a ellas, aunque podrían no estar exentas de otras "problemáticas psicosociales menores". Lo no problemático estaría dado por no escapar de una estructura tradicional de familia nuclear.

“...Hay familias que son impecables que tienen mamá preocupada, papá trabajador y los hijos..."

\subsection{Familias Problemáticas:}

Caracterizada según a) la organización familiar y b) el tipo de problemáticas psico-sociales.

Según la organización familiar se desprende:

1.2.1. Familias con una organización distinta a la Nuclear, señaladas como problemáticas por su conformación distinta a las familias nucleares, entre ellas se encontrarían: a) Familias extensas: Compuestas por más de un núcleo familiar; b) Familias articuladas en torno a la figura de la mujer/madre: Especialmente si esta tiene múltiples parejas.

“...Porque en una familia pueden haber dos o tres progenitores distintos, que es sumamente frecuente, dos hasta tres padres..." 
c) Familias en convivencia parcial: Núcleos familiares contingentes articulados en torno a la figura de la mujer/madre, en donde el hombre -aunque generalmente proveedor- transita en más de un núcleo familiar.

"Hay algunas mujeres que saben que algunos hombres tienen distintos hogares y se alojan una semana con ella y otra semana con la otra pareja, y ellas aceptan..."

\subsubsection{Familias con problemas psicosociales: La con-} dición de problemática estaría dada por la presencia de consumo abusivo de drogas y alcohol, embarazo adolescente, violencia y fundamentalmente pobreza.

“...Pero el real problema de violencia es el del hombre hacia la mujer, o sea el maltrato infantil es poco, o sea no es un porcentaje importante, mientras que la violencia del victimario digamos eso sí". "Y en cuanto a los niños hombres lo vemos como deserción escolar, abuso de alcohol o drogas eh... ingreso a pandillas, yo creo que por ahí va esta cosa que se da en la familia."

Esta calificación se asocia principalmente a familias provenientes de Santiago.

\section{Demandas de Atención Primaria.}

Da cuenta de las necesidades que motivan la demanda de las familias al sistema de salud. Respecto de estas demandas coincide la información relevada de las fichas con las percepciones de los equipos de salud en ambos estamentos de atención del CESFAM. Se distinguen dos tipos de necesidades fundamentales que subyacen a las demandas de atención: Necesidades de salud física/mental y necesidades psicosociales.

\subsection{Necesidades de Salud Física/Mental.}

2.1.1. Necesidades de Salud Física generalmente se corresponden a problemáticas asociadas al ciclo vital, demandas que son absorbidas por los Programas Niño Sano, Anticoncepción, Adulto Mayor entre otros.

2.1.2. Necesidades de Salud Mental, principalmente asociadas a trastornos depresivos, de ansiedad u otros desórdenes reactivos del ánimo (los trastornos mayores son derivados a la red asistencial secundaria).

\subsection{Necesidades Psicosociales:}

Los equipos las definen como aquellas que están fuera de sus competencias como CESFAM. Las valoran negativamente, en tanto producen una sobredemanda de atenciones, y dan cuenta de un mal uso de la red socialasistencial por parte de los usuarios. Estas problemáticas se desglosan en:
2.2.1. Pobreza, entendida como las dificultades en el acceso a la red social más amplia de satisfacción de necesidades básicas. Este fenómeno se relaciona con las características de la localidad y al difícil acceso de los recursos locales disponibles en la comuna y en la región a la que pertenecen.

“Es que aquí todo está cruzado por la pobreza, si esta comuna es muy pobre, entonces la gente anda contando la chaucha para la micro, para esto otro, entonces cualquier decisión familiar que se tome esta cruzado por el problema económico...y vienen para acá para que los ayuden"

2.2.2. Consumo de alcohol y drogas, asociado a las transformaciones que han ocurrido en los últimos años a nivel social en el país y en la localidad, lo que a juicio del equipo de salud se da por la migración de familias empobrecidas desde Santiago.

“... En los últimos años hemos tenido el problema del tráfico (...) hay varias familias que trafican (...) y nosotras sabemos más o menos quiénes son..."

2.2.3. Negligencia. Es una de las problemáticas a la que más relevancia dan los equipos de salud, generalmente se asocia a la función parental por la falta de información y preparación en conductas de cuidado doméstico en salud. Se sitúa en la responsabilidad de las mujeres el deber de poder realizar un reconocimiento prematuro de la enfermedad y de llevar a cabo prácticas de cuidado necesarias para evitar cuadros de gravedad mayor.

“...Acá la gente acude por un puro problema de negligencia de que no saben manejar las enfermedades en sus casas, por ejemplo llegan niños deshidratados por diarrea (...) hay bastante despreocupación”.

2.2.4. Embarazo adolescente. Es consignado como una de las problemáticas más recurrentes e importantes para los equipos de salud, quienes lo ven como un generador de problemas de salud, no sólo para la adolescente sino también para sus hijos y un estresor familiar.

"O sea mi prima quedó embarazá a los 15 años, mi mamá seguro que también, mi vecina también, entonces es parte de la subcultura, no importa todo es así y vamos teniendo hijos y nadie se hace mayor problema..."

Este tipo de demandas hacia el CESFAM también visibiliza las carencias en la cobertura de la red social y de salud presentes en la localidad. 
Figura 2

Relaciones y percepciones en los cuidados de salud: Producción de Salud Familiar y Atención del CESFAM

[A diferencia del esquema y categorías anteriores, esta categorización está construida exclusivamente sobre la información proveniente de las entrevistas a los equipos de salud. Se relevan las percepciones que, para estos equipos, tienen los cuidados domésticos en salud. Percepciones que articularían las relaciones usuarios/equipos en un espacio figurativo]

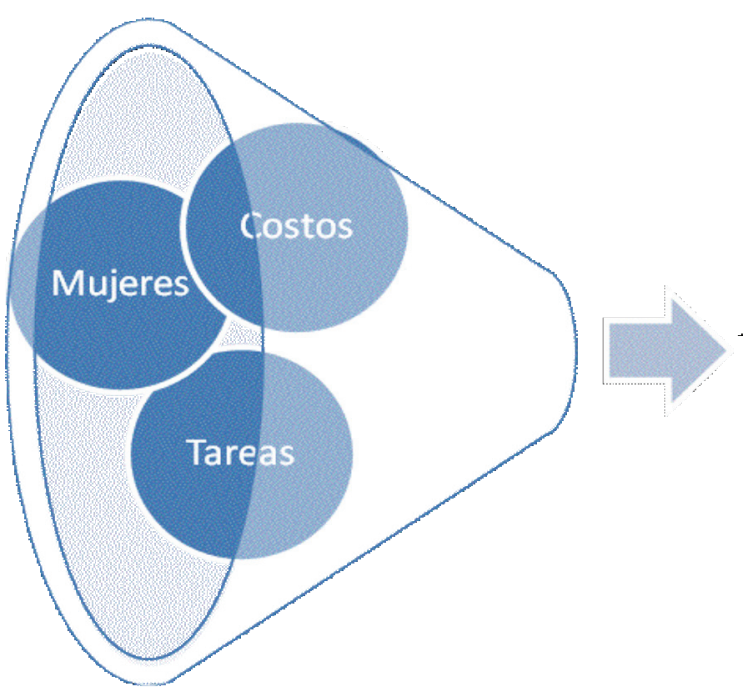

Para abordar la relación propuesta entre los cuidados domésticos en salud y las percepciones de los prestadores del sistema de salud respecto de las relaciones de los cuidados en las familias, se establecen las siguientes categorías.

\section{Organización de los Cuidados Domésticos en Salud:}

Categoría que señala quién ejerce los cuidados, los tipos de cuidados y los costos del cuidado de la familia. Se destaca que son las mujeres en su calidad de madres o abuelas (por lo general en edad reproductiva) quienes figuran como adulto responsable que encabeza el grupo familiar usuario y ocupándose de los niños, niñas y adultos mayores, que son los consultantes más frecuentes. En cambio el lugar de los hombres en la organización del cuidado se reduce a hacerse cargo de sí mismos cuando están aquejados por una enfermedad que interfiere de manera significativa con su vida cotidiana o laboral, siendo la mujer la organizadora de los cuidados incluso en esos casos.

\subsection{En el quién ejerce los cuidados...}

se observa que son las mujeres las que asumen los cuidados, ya sea como madres o abuelas (para el caso de los niños y niñas), como hijas (para el caso de los adultos mayores) o como esposas (en relación a las enfermedades de los cónyuges).

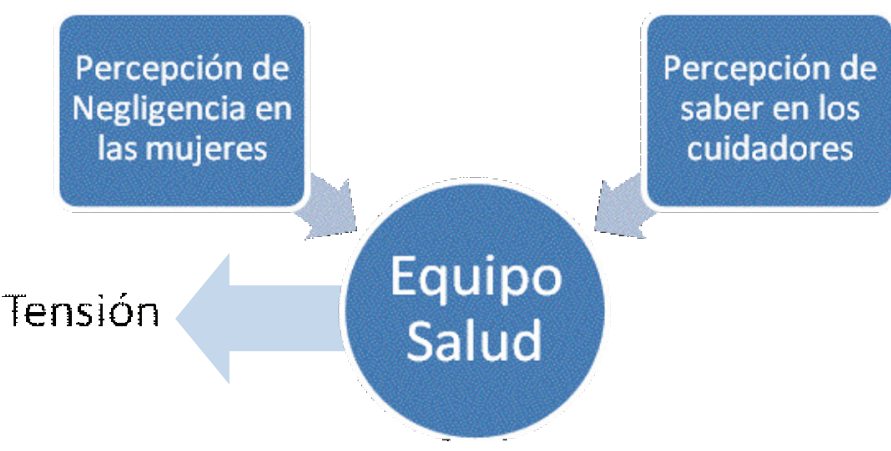

“...La mujer en general asume los cuidados, las dueñas de casa y las abuelas, muchas abuelas tienen un rol muy importante dentro de la familia..."

Además estos cuidados son generalmente invisibilizados y cuando se habla de ellos se los refiere en términos masculinos.

“... El pobre cuidador está con lumbago y muchas veces lo retan porque no le cambió la sonda y el pobre estaba cuncucho ese día, entonces pasan 100 mil detalles..."

De otra parte, los hombres asumirían secundariamente algunos roles como el de acompañante en situaciones muy especiales de salud o en el caso de embarazo de la pareja.

\subsection{Los tipos de cuidados,}

estarían clasificados en: cuidados de acompañamiento del enfermo o enferma a la consulta, cuidado doméstico, tareas de cuidado permanente en enfermedades crónicas, condiciones especiales de capacidad física y/o mental y adultos/as mayores.

“...Y es porque la madre se siente sobrepasada, o sea cuidando 10 cabros, con un marido como las huifas, que tiene que cuidar a la mamá que está hemipléjica..." 


\subsection{Impacto y Costos.}

Se refiere a los costos económicos, sociales y subjetivos que tienen las tareas de cuidado doméstico para las familias, particularmente para las mujeres y en el impacto en la calidad de vida de las cuidadoras. Los cuidados de los enfermos crónicos son los percibidos como los que generan mayor impacto y costos para las familias. Estos se relacionarían con: a) Costos que fluctúan entre permisos constantes y renuncia al trabajo. Continuo que impacta a las cuidadoras, deteriorando la estabilidad laboral y acrecentando la pobreza e influyendo en la vulnerabilidad psicosocial de las familias; b) Costos monetarios y de tiempo que implican el traslado, la compra de medicamentos y otros trámites propios del cuidado. c) Impacto psicológico y físico en la calidad de vida de las cuidadoras, pese a que los cuidados son ejercidos por mujeres, la idea del cuidador aparece masculinizada. Dicho impacto está expresado en cansancio emocional, estrés y malestares físicos generalizados.

“...A veces los cuidadores también llegan con un cuadro de depresión tremendo y no quieren saber nada más del mundo..."

2. Valoración del ejercicio de los cuidados domésticos en Salud.

Esta categoría está referida a la calificación valorativa con que los equipos de salud evalúan los cuidados en salud realizados en el espacio doméstico.

\subsection{Manejo básico de enfermedades:}

Da cuenta de la importancia que los equipos de salud adscriben a los tipos de manejos con que se enfrenta la enfermedad en el espacio doméstico. Se destaca que los equipos evalúan como deficientes e inadecuados los manejos frente a la enfermedad en las familias, lo que se relaciona con la idea de negligencia antes desarrollada.

“... Porque es obvio que el niño con una diarrea se va a deshidratar... entonces la mitad de los casos que llegan no deberían llegar si tomaran las precauciones necesarias al inicio de una enfermedad..."

\subsection{Fallas en las tareas de cuidados:}

Se refiere a las fallas en el funcionamiento apropiado en los cuidados básicos en las familias, previos a la enfermedad y/o a la consulta médica; a los modos de vida y a la inhabilidad de los cuidadores para proporcionar normas, hábitos y situaciones de salubridad básica y producción de la salud.

“...Uno ve en el consultorio que no cumplen con los controles de salud, en la vivienda eh...no tener los cuidados de mantener la casa limpia, no tener a los niños con ciertos hábitos eh...cuando los niños no quieren ir al colegio no los mandan no más eh...y también está asociado a este estilo de vida un tanto caótico que tienen estas familias de vivir..., si uno va a hacer visitas por ejemplo a las doce del día, uno podría esperar que la mamá o la abuela estuviera preparando el almuerzo, pero están tomando desayuno... entonces en eso se nota harto eso de la negligencia y de la creación de hábitos"

Se destaca que no existen valoraciones positivas en torno al ejercicio de los cuidados en el espacio doméstico.

3. Tensión socio-cultural, equipos de salud y familias usuarias:

Esta tensión es concebida en relación a una percepción de asimetría sociocultural en torno a la producción de la salud, que se constituye como un obstáculo para la misma.

“...Hay que hacer lo humanamente posible, pero es una subcultura que vive con patrones distintos a los nuestros, y es sencillamente así; entonces no es bueno, ni malo, son distintos..."

\section{Discusión}

El funcionamiento del CESFAM y la consiguiente implementación del Modelo de Salud Familiar, depende en definitiva de sus equipos y de las visiones que éstos tienen sobre la organización de las familias y el funcionamiento de las mismas, siendo en esto gravitante la construcción cultural e ideológica con que se conceptualizan a estos beneficiarios. Profundizamos de esta manera, en los discursos que construyen los equipos sobre los cuidados de la salud familiar.

A la luz de las tipificaciones familiares construidas a partir del análisis de las fichas y los discursos de los equipos de salud sobre las familias usuarias del CESFAM, es fácil de inferir que las principales depositarias de la responsabilidad de la salud familiar son las mujeres -que en su condición de dueñas de casa y/o trabajadoras asalariadas, en este caso con baja escolaridad- asumen desde el lugar de madres, hijas o abuelas, las demandas de atención de las familias. Situación que se visibiliza en que la gran mayoría de las fichas familiares tienen como adulto responsable a mujeres.

La responsabilidad de las mujeres en la producción de salud tiene como principal función el ejercicio de las tareas de cuidado de salud familiar, que se expresan en múltiples motivos de consulta y/o atención, como el control de la anticoncepción (propio o de las hijas), el control del embarazo, el cuidado de los hijos, hijas, nietos nietas y/o el cuidado de algún adulto mayor, lo que por lo demás, coincide con el estudio de Batthyány (2004).

Respecto de este protagonismo de las mujeres en relación a la salud familiar, los equipos de salud construyen un discurso, en torno a las familias, en que las mujeres sólo son tomadas en cuenta, en tanto objeto de cuestionamiento de la producción de salud familiar, por lo que los equipos de salud 
invisibilizan y distorsionan la centralidad del papel que ellas juegan en la relación de las familias con el CESFAM.

Esta distorsión en torno al papel de las mujeres en la salud en el hogar, articula la tipificación de las familias en dos perfiles característicos, las denominadas "familias problemáticas" y las "familias no problemáticas". Significaciones clasificatorias que dan cuenta de las representaciones hechas sobre las familias, las que se erigen en torno a ideas, tanto sobre la organización familiar, como al papel que juegan las mujeres en dicha organización. Así, para estos equipos una familia no-problemática es aquella con una organización nuclear donde la jefatura de hogar -a pesar de ser tramitada en la figura de una mujer- la ejerce un hombre. Esta figura masculina (aunque indirecta) aseguraría el supuesto de un mayor bienestar psicosocial y por tanto de salud; y por su ausencia se definiría a la familia problemática, la que alude a una organización sin presencia paterna y en contraste a esto, ubicando a las mujeres como gestantes de la problemática en la salud familiar, poniendo el acento en las fallas en los papeles tradicionales femeninos de reproducción de los cuidados e invisibilizando su asunción como jefas de hogar y por tanto como sostén económico.

Llama la atención de que independientemente de cómo se realizan los cuidados -ya que en ambos casos los realizan mujeres- es la presencia o ausencia de un hombre y su papel tradicional en la relación parental la que define la distinción entre familias problemáticas y no-problemáticas, considerando la presencia única de mujeres como una falla en su organización.

Especial énfasis, para la representación de una familia problemática, tiene la presencia de mujeres que han constituido familia con distintas parejas. Pareciera que esto podría deberse a la dificultad de articular -en relación al nacimiento de los hijos- una figura de padre organizadora de la familia y sus funciones. Al respecto cabe señalar que estas mujeres son significadas como "ejerciendo un tipo particular de prostitución", lo que además de aludir a la idea de "promiscuidad sexual" se referiría a la idea de "recurrir a varios proveedores".

Así podríamos inferir que la distinción sobre la problematicidad de las familias, se construye en el imaginario social de los equipos del CESFAM, por la presencia o ausencia de un padre/jefe, siempre mediado a través de cómo las mujeres logran instalar la figura del padre en el seno de la organización familiar. Cabe señalar una vez más que es en las mujeres en quienes recae la responsabilidad de construir una "familia adecuada" que preste soporte a las condiciones en que se enmarca el papel esperado de lo "femenino" en las tareas de cuidado. Por lo tanto, pensamos que los imaginarios de familias se construyen en un tipo de organización familiar articulado en torno al maternaje, ya que en dicho imaginario la depositaria "natural" del bienestar o problematicidad familiar es siempre la mujer.
Otro elemento definitorio de la condición problemática de la familia es "la problemática psicosocial", la que imprimiría la "disfuncionalidad" propia de dinámicas en que se destacan el consumo abusivo de drogas y alcohol, el embarazo adolescente, la violencia de género, las problemáticas juveniles y la pobreza.

Cabe señalar que a pesar de que la violencia de género aparece como una de las problemáticas psicosociales más frecuentes, ésta es nominada como violencia intrafamiliar, situación que siempre esconde el papel del hombre en el ejercicio de la violencia y obtura la responsabilidad masculina en la caracterización de familias problemáticas.

Respecto de las demandas de atención, categorías que dan cuenta de necesidades en salud física, salud mental y problemáticas psicosociales, son organizadoras de la condición problemática de las familias y son las que paradojalmente hacen presente la voz de las mujeres, que se traducen en un guión de circular reiteración: motivo de consulta-familia problemática. Así, son siempre las mujeres -vehiculizando problemáticas familiares- las que se ubican en el vértice de la tensión entre el sistema de salud y la producción del cuidado en la salud familiar.

Lo dicho nos permite postular que es siempre en la figura de las mujeres en donde recae y se reitera la condición de posibilidad de una familia disfuncional, siendo ellas el punto de convergencia de las demandas de los equipos a las familias y de las familias al sistema de salud.

En el mismo sentido, el embarazo adolescente-también relativo a las mujeres- es otro de los motivos frecuentes en la consulta del CESFAM. Se articula como disfuncional y propio de las familias problemáticas, y es parte del guión antes mencionado, que visibiliza una vez más a la disfuncionalidad de la familia como consecuencia del papel ejercido por la mujer, ya que influye en la generación de proyectos de vida tempranamente inscritos en su disfuncionalidad. Así, el embarazo adolescente dará reinicio al imaginario de que las mujeres perpetúan la problematicidad, ya que es la madre soltera y adolescente la que generará un nuevo proyecto de familia que casi seguramente será portador del sello de "familia problemática".

Desde un análisis de segundo orden (y en referencia al segundo esquema de categorías) habría que pensar las tareas de cuidado en lo que refiere a su organización social, como una perspectiva que muestra un continuo en el "ser mujer" construido desde su posición familiar, la que coincidentemente con lo planteado por Rodríguez (2005), produce y reproduce a lo femenino como lugar desde donde se organizan y ejercen los cuidados de salud de la familia.

Con relación a las tareas de cuidado cabe interrogarse por la posición desde donde se plantean las valoraciones acerca del ejercicio de estas en el espacio familiar. Siendo el sujeto de enunciación de las exigencias valorativas, el sistema de salud por medio de sus equipos profesionales, se puede señalar que dichas exigencias aparecen institucionalizadas por 
estos gracias a la apropiación del saber materno, que pasa a articular el saber implícito de las familias en la producción de las tareas de cuidado (Foucault, 2003; Vilchez, 2001). Consistentemente con esto los equipos de salud, como representantes del saber institucional-profesional de salud, también están compuestos en su mayoría por mujeres, es decir, la idea de este saber materno es así reproducido por las mujeres trabajadoras de la salud (médicas, enfermeras, psicólogas, paramédicas, personal administrativo, etc.); por tanto la apropiación del saber materno se practica también desde el papel de "cuidadoras profesionales", y se articula en el espacio de organización social del CESFAM en torno a exigencias y normas de control social e ideológico, perfilando la cotidianidad familiar producida alrededor de la mujer, la que es sostenida en la proyección de un ideal del yo, que no es otro que el ideal del ser mujer en los discursos patriarcales, es decir, un ser para otro.

En definitiva, los equipos de salud con su visión tradicional sobre la familia, presuponen que los cuidados se deben producir "naturalmente" en la propia familia y deben ser vehiculizados por las mujeres, quienes son vistas como portadoras de un saber implícito suficiente para la producción de salud y la interrelación con los equipos del CESFAM. Generándose una tensión no resuelta entre las pretensiones de modernización del sistema de salud y las concepciones de los equipos de salud en torno a las familias a las que esta modernización está dirigida. Resolución que es inviable, si se deja en este binomio de particular tensión atrapadas a las mujeres, las familias y los equipos de salud en pretensiones modernizantes que no hacen más que reproducir ideas que perpetúan lugares comunes en torno al género, la división sexual del trabajo y la organización familiar y que finalmente impactan negativamente en la calidad de vida y salud mental de las familias y las mujeres.

La tensión producida entre las demandas de atención de las familias -agenciadas por las mujeres-y la valoración negativa que construyen los equipos en torno a la producción de salud en el espacio doméstico, resulta un obstáculo para el éxito de la implementación del Modelo de Salud Familiar. Ya que los equipos de salud en cuestión, no logran incorporar una comprensión de los considerables cambios y las nuevas diversidades de las familias chilenas, menos aún las diversidades de las actuales configuraciones de género, hipotecando así la relación con quienes producen saludenfermedad y son la base social de la implementación del nuevo Modelo de Salud Familiar, es decir, las mujeres.

En relación a los cuidados domésticos en salud, podemos concluir que son las mujeres las que una vez más ejercen dichas tareas, desde sus papeles de hijas, madres y abuelas, constituyéndose éstas en la "base social necesaria" para llevar a cabo la implementación de la reforma del Sistema de Salud y del Modelo de Salud Familiar (Medel, Díaz \& Mauro, 2006).
Esta investigación muestra que sigue existiendo un vacío considerable entre lo que se sabe de los cambios de las familias y el papel de las mujeres en la vida social, y la comprensión que hacen los equipos de salud en torno a las familias y las mujeres, es decir, no existe la traducción de las comprensiones modernizantes a las prácticas modernizadoras que llevan a cabo los representantes del sistema de salud frente a las familias usuarias del sistema.

\section{Referencias}

Arriagada, I. (2005). Transformaciones sociales y demográficas de las familias latinoamericanas. En X. Valdés \& T. Valdés (Eds), Familia y vida privada ¿Transformaciones, tensiones, resistencias o nuevos sentidos? Santiago: CEDEM.

Batthyány, K. (2004). Cuidado infantil y trabajo: ¿Un desafío exclusivamente femenino?; Una mirada desde el género y la ciudadanía social. Montevideo: CINTERFOR.

Berlagoscky, F., \& Provoste, P. (2002). Modelo de atención y género: Las condiciones socioculturales de la reforma de la salud en Chile. Serie: Género, equidad y reforma de la salud en Chile, $\mathrm{N}^{\circ} 4$, Organización Panamericana de la Salud - Organización Mundial de la Salud. Proyecto Género, Equidad y Reforma de la Salud. Santiago.

Brito, P. (2000). Impacto de las reformas del sector salud sobre los recursos humanos y la gestión laboral. Revista Panamericana de Salud Pública, 8, 43-54.

Calvin, M.E., \& Grandon, A. (1995). Monitoras de Salud: Trayectorias de participación. Santiago: EPES.

CEPAL (2007, Agosto). El aporte de las mujeres a la igualdad en América Latina y el Caribe. X Conferencia Regional sobre la Mujer de América Latina y el Caribe, Quito, Ecuador

Di Gropello, E., \& Cominetti, R. (Edts). (1998). La descentralización de la educación y la salud. Un análisis comparativo de la experiencia latinoamericana. Santiago: CEPAL.

Durán, M. A. (2005). Los costes invisibles de la enfermedad. Madrid: Fundación BBVA.

Foucault, M. (2003). El nacimiento de la clínica: Una arqueología de la mirada médica. Buenos Aires: Siglo XXI.

Gálvez, T., \& Matamala, M. (2002). La economía de la salud y el género en la reforma de salud. En Género, equidad y reforma de la salud en Chile. Voces y propuestas desde la sociedad civil (1-7). Santiago: OPS-MINSAL.

González, N., \& Flores B. (2008). Políticas públicas y salud en América Latina. Elementos para una crítica a la política social neoliberal. San José, Costa Rica: ALACIP

Hopenhayn, M. (2009, octubre). Familias: Igualdad de derechos y derecho a la igualdad. Presentación en seminario "Las familias latinoamericanas interrogadas. Hacia la articulación del diagnóstico, la legislación y las políticas", Santiago, Chile.

Infante, A., Mata, I., \& López-Acuna, D. (2000). Reforma de los sistemas de salud en América Latina y el Caribe: Situación y tendencias. Revista Panamericana Salud Pública, 8, 13-20. Recuperado en Noviembre, 14, 2009 disponible en http://www.scielosp.org/scielo.php?pid=S102049892000000700005\&script=sci_arttext

La Parra, D. (2001). Contribución de las mujeres y los hogares más pobres a la producción de cuidados de salud informales. Revista Gaceta Sanitaria, 15, 498-505.

Medel, J., Díaz, X., \& Mauro, A (2006). Cuidadoras de la Vida: Visibilización de los costos de la producción de salud en el hogar. Impacto sobre el trabajo total de las mujeres. Documento de trabajo CEPAL.

Mesa-Lago, C (2005). Las reformas de salud en América Latina y el Caribe: Su impacto en los principios de la seguridad social. Documentos de proyectos CEPAL 
MINSAL (2002). Los Objetivos sanitarios para la Década 2000-2010. Dirección de Rectoría y Regulación Sanitaria / Departamento de Epidemiología. Santiago.

MINSAL (2008). Orientaciones para la programación en red. Subsecretaria de Redes Asistenciales / División de Gestión de la Red Asistencial / División de Atención Primaria. Santiago.

Olavarria, J. (2001). Hombres a la deriva: Poder, trabajo y sexo. Santiago: FLACSO.

Provoste, P. (2002). Género, equidad y reforma de la salud en Chile participación en la Salud Pública: Una aproximación de Género. Serie Género y Reforma de la Salud. Santiago: OPS/OMS Chile.

Reca, I., Álvarez, M., \& Tijoux, M.E., (2008). Costos no visibles del cuidado de enfermos en el hogar: Estudio de casos en Chile. En La economía invisible y las desigualdades de género, La importancia de medir y valorar el trabajo no remunerado (179-194). Washington D.C.: OPS.

Rico, N. (2009, octubre). Crisis de cuidado y políticas públicas, El momento en ahora. Presentación en seminario "Las familias latinoamericanas interrogadas. Hacia la articulación del diagnóstico, la legislación y las políticas", Santiago, Chile.
Rodríguez, A (2005). Estrategias en los cuidados y relaciones de género: Aproximaciones desde la antropología. Granada: Instituto de estudios de la mujer.

Ugalde, A., \& Homedes, N. (2002), Descentralización del sector salud en América Latina. Revista Gaceta Sanitaria, 16, 18-29. Recuperado en Noviembre 6, 2009 disponible en http://www.doyma.es/revistas/ ctl_servlet?_f=7064\&ip=66.249.71.65\&articuloid $=13026050$

UNIFEM-CEPAL (2005). Encuestas Nacionales sobre el Uso del Tiempo: Su diseño y aplicación. Santiago: OPS-UNIFEM-CEPAL.

Vilches Norat, V. (2001), De(s) madres o el rastro materno en las escrituras del yo: A propósito de Jacques Derrida, Jamaica Kincaid, Esmeralda Santiago y Carmen Boullosa. Santiago: Cuarto Propio. 